\title{
THE FORTY-NINTH REGULAR MEETING OF THE SAN FRANCISCO SECTION
}

The forty-ninth regular meeting of the San Francisco Section of the Society was held at the University of Washington on Saturday, June 12, 1926. In the absence of the Chairman, Professor Blichfeldt, Professor Daniel Buchanan was elected temporary Chairman. The total attendance was twenty, including the following fifteen members of the Society:

E. T. Bell, Bernstein, Daniel Buchanan, A. F. Carpenter, Cramlet, Dines, Gavett, Hanawalt, Jerbert, McAlister, W. E. Milne, Mullemeister, Neikirk, Rojansky, Winger.

Following the meeting, the members of the Section at the University of Washington acted as hosts to the visiting members and their friends at a luncheon and an excursion on Puget Sound.

Abstracts of the papers presented at this meeting follow below. The papers by Brown, Buchanan (first paper), Cleveland, Gage, Gehman, Johnston, D. H. Lehmer, D. N. Lehmer, and Whyburn were read by title. Mr. Brown, Mr. Gage, and Miss Johnston were introduced by Professor Buchanan, and Mr. Cleveland by Professor R. L. Moore.

1. Professor E. T. Bell: The modular Bernoullian and Eulerian functions.

This paper, to appear shortly in the publications in science of the University of Washington, is a brief prospectus concerning a remarkable new class of polynomials in two complex variables. In one of the complex variables they are Appell polynomials; in the other they satisfy no similar algebraic differential equation. The theory includes as very special cases those of the Bernoulli and allied numbers, the anharmonic polynomial generalizations of these devised by the author, and the common Bernoullian and Eulerian functions. The general theory is more readily developed than is that of any of its special cases, because in the latter the underlying symmetry, which is that of the linear group abstractly identical 
with the cross-ratio group, is obscured owing to coincidences of two or more values of the complex variables at the same point.

\section{Professor E. T. Bell: Irregular fields.}

An irregular field $I F$ differs from an abstract field $F$ only in that division by $m>1$ "irregular elements" in $I F$ is not permissible (instead of the unique zero element in $F$ ). The postulates for $I F$ are stated in abstract form and then solved, giving several interconnected $I F$ 's. Finally, the abstract theory is applied to the algebra of the elliptic polynomials-a new class of polynomials including as degenerate cases the Bernoullian, Eulerian, and their anharmonic generalizations. Differential and difference equations in $I F$ are discussed and applied.

3. Professor E. T. Bell: On a fundamental theorem in the theory of class-number relations.

This paper will appear in full in an early issue of this Bulletis.

4. Professor B. A. Bernstein: On the serial relation in Boolean algebras.

This paper appears in full in the present issue of this Bulletin.

5. Miss C. I. Johnston: Isosceles-triangle solutions when the two finite bodies are oblate spheroids.

When two finite spheres move in circles about their common center of gravity, an infinitesimal third body will describe a periodic orbit, if given the proper initial projection. This orbit is the straight line through the center of gravity of the finite bodies and perpendicular to the plane of their motion. This solution is called the isosceles-triangle solution. The present paper determines the corresponding isosceles-triangle solution when the finite bodies are similar oblate spheroids of equal mass. It will appear in the Transactions of the Royal Society of Canada.

6. Mr. J. F. Brown: Asymptotic orbits near the straightline equilibrium points in the problem of three bodies when two of the bodies are oblate spheroids.

Warren and Strömgren determined, independently, the orbits which are asymptotic to the straight-line equilibrium points, when two of the bodies are finite spheres moving in circles, and the third body is infinitesimal. This paper deals with the corresponding orbits when the finite bodies are oblate spheroids. It will appear in the next issue of the TranSACTIONS OF THE ROYAL SOCIETY OF CANADA.

7. Professor Daniel Buchanan: Isosceles-triangle orbits of the second genus.

Poincare has shown the existence of two types of periodic orbits in the problem of three bodies. These he called orbits of the first and of the second 
genus. If the first-genus orbits have the period $T$, then the second-genus orbits will have the period $k(T+p)$, where $k$ is an integer and $p$ is a parameter. This paper deals with the second-genus orbits near one of the isosceles-triangle solutions previously determined by the author; it will appear in the Transactions of the Royal Society of Canada.

8. Professor Daniel Buchanan: Periodic orbits of the second genus near the straight-line equilibrium points.

Moulton's "oscillating satellite" exhibits periodic orbits near the straight-line equilibrium points in the problem of three bodies. These orbits are of the first genus. This paper determines the second-genus orbits in the vicinity of Moulton's solutions. Along with the preceding paper, it is the first to exhibit orbits of the second genus.

9. Mr. W. H. Gage: An illustrative orbit of the second genus.

The earlier steps of the integrations in the preceding paper are carried out algebraically. The approximate second-genus orbits are determined for certain assigned values of the masses, initial displacements, etc.

\section{Professor A. F. Carpenter: Intersector sequences.}

On each of an ordered set of three ruled surfaces $R_{1}, R_{2}, R_{3}$, whose rulings $r_{1}, r_{2}, r_{3}$ correspond in sets of three, one from each surface, there lies a one-parameter family of curves whose tangents, called intersector tangents of the surface, are such that those tangent to $R_{1}$, along $r_{1}$, cut $R_{2}$ along $r_{2}$, those tangent to $R_{2}$ along $r_{2}$ cut $R_{3}$.along $r_{3}$, etc. The paper defines certain properties of sequences of these intersector tangents and establishes a theorem involving a general invariant.

11. Professor A. F. Carpenter: Closed systems of invariants in projective differential geometry. Preliminary communication.

A system of ten invariants in the projective differential theory of triads of ruled surfaces is shown to be closed with reference to two different operations.

12. Mr. C. M. Cramlet: Applications of the determinant and permanent tensors to determinants of general class and allied tensor functions.

With a given set of fundamental tensors, tensor functions can be expressed by the tensor $\gamma_{s_{1} \ldots s_{n}}^{r_{1} \ldots r_{n}}$ (see this BulletiN, vol. 32, p. 213). Determinants and permanents are examples of such functions. Theorems for permanents formally analogous to the theorems for transformations and multiplication of determinants of a rank 2 tensor are given. By the use of 
the gamma tensor and its specializations the determinant and permanent tensors, insight into the properties of determinants of higher class is obtained. In particular, the rule for file multiplication appears to be applicable to more general types of functions than have been considered heretofore.

\section{Professor L. L. Dines: Note on certain associated} systems of linear equalities and inequalities.

In this note the author presents some theorems complementary to those of his paper in the Annals of Mathematics (1925).

\section{Professor L. L. Dines: General systems of vectors.}

A set of $m$ real numbers $\left(a_{1}, a_{2}, \cdots, a_{m}\right)$ may be interpreted as the components of a vector in space of $m$ dimensions. A set of $m$ real functions $\left(f_{1}(p), f_{2}(p), \cdots, f_{m}(p)\right)$ of a variable $p$ may therefore be interpreted as a system of vectors, each value of the parameter $p$ determining a vector of the system. In the present paper the author considers certain properties of a general system of vectors, the generality consisting in the fact that the range of the parameter $p$ is entirely unconditioned. The methods and results generalize some of those contained in earlier papers of the author.

15. Professor L. L. Dines: On linear integral inequalities.

This investigation is a result of an effort to generalize to functions on a general range, the theory developed by the author for systems of linear algebraic inequalities. Peculiar difficulties are inherent in the case of continuous functions of a continuous variable, and it therefore seemed best to consider this case alone. The study of the integral inequality $\phi(x)-\int_{0}^{1} \alpha(x, t) \phi(t) d t>0$ involves the study of the functions orthogonal to a given set of functions $\psi_{1}(x), \psi_{2}(x), \cdots, \psi_{n}(x)$, in particular the conditions under which such a function may be everywhere positive.

16. Mr. C. M. Cleveland: Concerning cut points of a continuous curve.

The author establishes the following theoems: I. Every connected set of cut points of a continuous curve is arcwise connected. II. Every maximal connected set of cut points of a continuous curve is an acyclic continuous curve.

17. Dr. H. M. Gehman: Some relations between a continuous curve and its subsets.

In this paper, the author proves that if $M$ is a continuous curve, a necessary and sufficient condition that every sub-continuum of $M$ be a continuous curve, is that every irreducibly connected set between two points of $M$ is an arc. Either of the following conditions is sufficient in order that every subcontinuum of a continuum $M$ be a continuous curve: (1) given any positive number $e, M$ contains at most a finite number of 
mutually exclusive continua of diameter greater than $e$; (2) given any positive number $e, M$ can be expressed as the sum of a finite number of continua each of diameter less than $e$, and each pair having at most a finite number of points in common. It is shown that certain other conditions which are sufficient when $M$ is assumed to be a continuous curve, are not sufficient under the weaker assumption that $M$ is a continuum.

18. Mr. D. H. Lehmer: Note on solving the indeterminate equation $t^{2}-p^{2} D u^{2}=1$.

The solution of the Pellian equation in which the argument $p^{2} D$ contains a square factor can be conveniently obtained from the solution of the equation for the argument $D$. The method depends on discovering among the multiple solutions of the latter equation, one in which the $u$ is divisible by $p$. Several theorems are given which limit the number of trials in searching for this solution. The simple case in which $p=2$ seems to have been noticed by Legendre. This method would be useful in extending the tables of the solutions of the Pellian equation, since it obviates the necessity of expanding the square root of the argument in a continued fraction.

19. Mr. D. H. Lehmer: Note on the Mersenne number $2^{139}-1$.

This paper appears in the present issue of this BuLLETIN.

20. Professor D. N. Lehmer: Further results in the congruential theory of magic squares. Second preliminary paper.

The author has reduced the theory of uniform step magic squares (odd order) to the theory of two simultaneous congruences involving the greatest integer function. These congruences involve essentially four arbitrary constants, and the various kinds of magic squares are obtained by subjecting these constants to certain conditions. His latest results are connected with nuclear squares.

21. Professor L. I. Neikirk: Some finite linear nonassociative algebras. Second paper.

The present paper is a generalization and extension of a previous paper on the same subject (see this BulLetro, vol. 19, p. 195). A complete set of postulates is given and a development of the general theory. Three kinds of powers are possible: $(S(\cdots(S(S(S S))) \cdots)),(\cdots(((S S) S, \cdots)$ $S),(\cdots((S(S S)) S) \cdots)$. Two examples of these algebras are exhibited along with multiplication tables. Both papers will be published in THE University of Washington Studies, mathematical series, number 3.

22. Mr. G. T. Whyburn: Concerning the cut points and end points of continua.

If $K$ and $H$ denote the set of all the cut points and end points respectively of a continuum $M$, then (1) every bounded subcontinuum of 
$K+H$ is an acyclic continuous curve; (2) if $T$ is a subcontinuum of $K$, and $Z$ is any connected subset of $M-T$, then $T$ contains at most one limit point of $Z$; (3) every subcontinuum of $K$ contains a subcontinuum belonging to the boundary of some single complementary domain of $M$; (4) $M$ is connected im kleinen at every point of $H$ which is accessible from $S-M$; (5) if $M$ is irreducible between some pair of points, then it is connected im kleinen at every point of $H$; (6) no point of $H$ is a boundary point of more than one complementary domain of $M ;(7)$ in order that a point $P$ of $M$ should belong to $K$, it is sufficient that it should be a cut point of the boundary of some complementary domain of $M$; (8) a point of a continuous curve $M$ is an end-point if it is not interior to any arc of $M$.

\section{Mr. G. T. Whyburn: Concerning continuous curves.}

R. L. Moore has shown that a continuous curve $M$ is an acyclic continuous curve if and only if $M$ is not disconnected in the weak sense by any subset of all the non-cut points of $M$. The author shows that this proposition holds for any bounded continuum. It is also shown that if $K$, $H$, and $N$ respectively denote the set of all the cut points, end points, and simple closed curves of a continuous curve $M$, then (a) $H$ is totally disconnected; (b) $K+H+N=M$; (c) $K N$ is countable; (d) if $T$ is any connected subset of $K$, then $\bar{T}$ is an acyclic continuous curve if and only if $T$ belongs to $K+H$; (e) $K$ does not contain more than a finite number of mutually exclusive continua of diameter greater than a given positive number; (f) if $L$ is any connected subset of $M-N$, then $L$ is arcwise connected and $\bar{L}$ is an acyclic continuous curve; (g) if the point $P$ belongs to $M$ but not to the boundary of any complementary domain of $M$, then $M$ contains a simple closed curve enclosing $P$ and of diameter arbitrarily small. (The italicized portion of (g) was established by C. M. Cleveland.)

\section{Professor E. H. McAlister: A new method of deter-} mining azimuth.

In contrast with existing methods, the peculiar merit of the method described is that it does not require any knowledge either of the latitude or local time. The observations and computations are both simple and brief. The accuracy attainable is nearly the same as the accuracy with which the observer can measure the horizontal angle between Polaris and the azimuth mark, all other small errors of observation being reduced in the ratio of one to thirty or better. Certain quantities, derived from the apparent positions of the stars, have been tabulated at ten-day intervals for the quarter century 1925-1950. The method is available at any season, at any hour of darkness, and in any latitude in which Polaris can be conveniently observed. The method has been generalized, with some loss of simplicity in computation, but with greater freedom in the choice of stars.

$$
\begin{aligned}
& \text { B. A. BERnStEIN, } \\
& \text { Secretary of the Section. }
\end{aligned}
$$

